\title{
Comparative Study of Microscope Assisted Tympanoplasty and Endoscope Assisted Tympanoplasty
}

\author{
Original Rigzing. C. Dadul', Deviprasad Dosemane ${ }^{2}$, Panduranga. M. Kamath ${ }^{3}$, Suja. S. \\ Article Sreedharan ${ }^{4}$, Vijendra Shenoy ${ }^{5}$ \\ ${ }^{1}$ Consultant ENT surgeon, Gangtok, Sikkim, 2,3,4,5 Department of Otorhinolaryngology, \\ Kasturba Medical College, Mangalore, Manipal Academy of Higher Education, India.
}

\begin{abstract}
Aim: Endomeatal endoscopic tympanoplasty (ET) is a relatively new technique. We wanted to study the feasibility of performing this technique, its outcome using tragal perichondrium (TP) and its cosmetic benefits over the conventional microscopic tympanoplasty (MT) using temporalis fascia (TF).

Patients and Methods: Study consisted of 81 patients in total between the age group 7 years to 70 years who fulfilled the inclusion criteria. Of these patients, 41 underwent MT using TP, while the remaining 40 underwent ET using TP as graft material.

Results: For the graft uptake and hearing gain, ET and MT had similar outcomes, but time taken for surgery for ET was significantly less than the time taken for MT. In addition to this, satisfaction of clarity of view, depth perception, round window reflex, and ossicular chain visualization were all comparable in both groups but the visualization of the entire tympanic membrane was better in ET group. Cosmetic satisfaction of ET were significantly better than the MT group. Conclusion: Endoscope has a better visualization of the entire tympanic membrane without the need to manipulate the patients head. ET was an equally effective technique and TP was an efficient graft material. Conventional MT required a long post auricular incision, dissection and closure which lead to change in normal orientation of the pinna leading to poor cosmetic satisfaction. The dissection and closure also extended the duration of surgery which was not required in endoscopic technique which lead to significant reduction of duration of surgery and better cosmesis.
\end{abstract}

Key Words: Chronic suppurative oOtitis media, cosmesis, endoscope, microscope, tympanoplasty.

Received: 31 December 2019, Accepted: 24 April 2020

Corresponding Author: Deviprasad Dosemane, MD, Associate professor, Department of Otorhinolaryngology, Kasturba Medical College, Mangalore, Manipal Academy of Higher Education. India, Tel.: +91-990135975, E-mail: dr.deviprasad@yahoo.com

ISSN: 2090-0740, November 2020 Vol.21, No.3

\section{INTRODUCTION}

Soon after the advent of the endoscope, it found its way into ENT surgeries and is currently being used for various complex ear surgeries. However, the microscope has always been an essential armament in an ENT surgeon's arsenal ${ }^{[1]}$. Till date, many discussions have sprung up over the years comparing the endoscopic technique to the microscopic one, the most encountered one being the comparison of Endoscopic Tympanoplasty (ET) and Microscopic tympanoplasty $(\mathrm{MT})^{[2]}$. In these techniques, i.e. the ET and MT, various outcomes that were investigated were uptake of the graft, improvement in hearing and duration of surgery ${ }^{[3]}$.

\section{AIM OF THE STUDY}

Our study aimed to compare the microscope assisted post auricular approach tympanoplasty and endoscope assisted endomeatal tympanoplasty, among patients with CSOM.

\section{PATIENTS AND METHODS:}

\section{1- Study setting:}

This study was carried out in our tertiary care hospitals

\section{2- Study design:}

Hospital-based cohort study.

\section{3- Study population:}

Patients presenting to our outpatient department, diagnosed as having Chronic Suppurative Otitis Media (CSOM) mucosal type and who were planned for tympanoplasty were considered for the study. A total of 81 patients were selected, out of which 40 patients (group A) underwent endoscopic tympanoplasty (ET) while 41 patients underwent microscopic tympanoplasty (MT). 


\section{4- Inclusion Criteria:}

Patient with CSOM mucosal type with dry ear for a minimum period of three weeks and age group between 7-70 years.

\section{5-Exclusion Criteria:}

CSOM squamosal type, actively discharging ear, only good hearing ear.

\section{6- Sampling strategy /method:}

Convenient sampling (non-random) was used to recruit the study participants.

\section{7- Study duration:}

Period of two years.

\section{8-Data collection methodology:}

The protocol was submitted for approval to the Institutional Ethical Committee (I.E.C) of our university and after obtaining approval, necessary permission was taken from the authority of the study hospitals. The hospitals were visited for data collection, and eligible patients were approached to be part of the study and explained about the objectives, in the language best understood by them. Anonymity was assured, and patients were given a participation information sheet. A written and informed consent was taken from each patient. Using the study pro forma, preoperative data was collected which included information on demography, clinical details and postoperative data.

\section{9- Statistical analysis:}

Chi square test was used along with student $\mathrm{T}$ test to compare across the groups. $P$ value of $<0.05$ was considered as statistically significant.

\subsection{0-Pre-operatively:}

Pure Tone Audiometry (PTA) was done to assess the degree and type of the hearing loss. The guidelines outlined by the "American speech and hearing association (ASHA)"[4] were used for doing PTA. Air bone(A-B) gap for each ear were noted separately, for frequencies $500 \mathrm{~Hz}$, $1 \mathrm{KHz}$ and $2 \mathrm{KHz}$, and the loss in hearing was calculated by taking the average of the three ${ }^{[5]}$.

\subsection{1- Intraoperatively:}

Difference in intraoperative findings between the two techniques was assessed in respect to satisfaction of clarity of view, satisfaction of depth perception (which was graded as Average or Good), whether entire tympanic membrane was visualised without need for manipulation of patient's head (evaluated as Yes or No), ossicular chain visualization and round window reflex elicitation (graded as Easy or Difficult) and finally duration of the surgery ${ }^{[6]}$.

\subsection{2- Post-operatively (3 months follow up):}

Graft uptake was confirmed by otoscopic findings, and PTA was done to assess the improvement in hearing ${ }^{[7]}$. Patient's cosmetic satisfaction (scar) was assessed by subjective analysis: patients were asked to grade the scars postoperatively. Patients were asked if numbness of the pinna/tragus (operated ear) was present and was subsequently graded. Similarly, pain over the incision, change in orientation of pinna/tragus and overall cosmetic satisfaction were also assessed and graded ${ }^{[8]}$.

\subsection{3-Surgical Technique:}

ET was done by endomeatal approach and the MT was done by postaural approach. The graft used for the endoscopic group was tragal perichondrium while for the microscopic group was temporalis fascia. In both the groups graft placement was by underlay technique.

\section{RESULTS:}

\section{1- Patient Demographics:}

There was a total of 81 patients included in our study, diagnosed to have CSOM mucosal type from September 2016 to August 2018. Out of these, 40 patients underwent ET with tragal perichondrium (TP) graft and 41 patients underwent conventional MT with temporalis fascia (TF) graft.

Age- The minimum age in the case group was 15 years and the maximum was 66 years with an average age of 40.5 years, whereas in the control group minimum age was 16 years and the maximum was 48 years with an average age of 32 years.

Gender- In ET there were 22 (55.0\%) females and 18 $(45.0 \%)$ males, while in MT there were $23(56.1 \%)$ females and $18(43.9 \%)$ males.

\section{2- Examination findings:}

On examination in ET group, 20 (50\%) patients were found to have a large central perforation (CP), $14(35 \%)$ patients were found to have medium $\mathrm{CP}$ and $6(15 \%)$ were found to have small CP. In MT group, $8(19.5 \%)$ patients were found to have large CP, 17 (41.5\%) patients were found to have medium $\mathrm{CP}, 9(22.0 \%)$ patients had small $\mathrm{CP}$ and $7(17.1 \%)$ had subtotal perforations. 


\section{3- Intraoperative findings:}

These are described in (Table 1).

\section{4- Duration of Surgery:}

In ET group, out of 40 cases, $36(90.0 \%)$ cases took 1 hour and below while 4 cases took more than 1 hour (10\%). Minimum time taken for surgery was 45 min, maximum time taken was $1 \mathrm{~h} 20 \mathrm{~min}$ and average time taken was $62.5 \mathrm{~min}$. On the other hand, in the MT group, 2 (4.9\%) cases took 1 hour or less and 39 (95.1\%) cases took more than 1 hour. The minimum amount of time taken was 1-hour, maximum time was $2 \mathrm{~h} 30 \mathrm{~min}$ and average time was $1 \mathrm{~h} 55 \mathrm{~min}$. The $\mathrm{p}$ value was highly significant $(p=0.00)$.

\section{5-Audiological evaluation:}

Details are in (Table 2).

In our study we were unable to compare the outcomes of Eustachian tube function and its effect on graft take up, as the majority of our patients had normal ETF.

\section{6- Post-operative cosmesis:}

Details are in (Table 3).

Table 1: Intraoperative findings

\begin{tabular}{|c|c|c|c|c|c|c|c|}
\hline & & \multicolumn{2}{|c|}{ Case } & \multicolumn{2}{|c|}{ Control } & \multicolumn{2}{|c|}{ Total } \\
\hline \multirow{3}{*}{$\begin{array}{l}\text { Satisfaction of } \\
\text { clarity of view }\end{array}$} & Average & 1 & $2.5 \%$ & 0 & $.0 \%$ & 1 & $1.2 \%$ \\
\hline & Good & 39 & $97.5 \%$ & 41 & $100.0 \%$ & 80 & $98.8 \%$ \\
\hline & Total & 40 & $100.0 \%$ & 41 & $100.0 \%$ & 81 & $100.0 \%$ \\
\hline \multirow{3}{*}{$\begin{array}{l}\text { Satisfaction } \\
\text { of depth } \\
\text { perception }\end{array}$} & Average & 3 & $7.5 \%$ & 5 & $12.2 \%$ & 8 & $9.9 \%$ \\
\hline & Good & 37 & $92.5 \%$ & 36 & $87.8 \%$ & 73 & $90.1 \%$ \\
\hline & Total & 40 & $100.0 \%$ & 41 & $100.0 \%$ & 81 & $100.0 \%$ \\
\hline \multirow{3}{*}{$\begin{array}{l}\text { Entire } \\
\text { tympanic } \\
\text { membrane } \\
\text { visualisation }\end{array}$} & No & 3 & $7.5 \%$ & 23 & $56.1 \%$ & 26 & $32.1 \%$ \\
\hline & Yes & 37 & $92.5 \%$ & 18 & $43.9 \%$ & 55 & $67.9 \%$ \\
\hline & Total & 40 & $100.0 \%$ & 41 & $100.0 \%$ & 81 & $100.0 \%$ \\
\hline \multirow{2}{*}{$\begin{array}{l}\text { Round } \\
\text { window reflex }\end{array}$} & Easy & 40 & $100.0 \%$ & 41 & $100.0 \%$ & 81 & $100.0 \%$ \\
\hline & Total & 40 & $100.0 \%$ & 41 & $100.0 \%$ & 81 & $100.0 \%$ \\
\hline \multirow{3}{*}{$\begin{array}{l}\text { Ossicular } \\
\text { chain } \\
\text { visualization }\end{array}$} & Difficult & 1 & $2.5 \%$ & 4 & $9.8 \%$ & 5 & $6.2 \%$ \\
\hline & Easy & 39 & $97.5 \%$ & 37 & $90.2 \%$ & 76 & $93.8 \%$ \\
\hline & Total & 40 & $100.0 \%$ & 41 & $100.0 \%$ & 81 & $100.0 \%$ \\
\hline
\end{tabular}

Table 2: Audiological Evaluation.

\begin{tabular}{llcccccc}
\hline & \multicolumn{2}{c}{ Case } & \multicolumn{2}{c}{ Control } & \multicolumn{2}{c}{ Total } \\
\hline \multirow{2}{*}{$\begin{array}{l}\text { Pre-operative } \\
\text { hearing }\end{array}$} & Mild & 25 & $62.5 \%$ & 23 & $56.1 \%$ & 48 & $59.3 \%$ \\
& Moderate & 15 & $37.5 \%$ & 18 & $43.9 \%$ & 33 & $40.7 \%$ \\
& Total & 40 & $100.0 \%$ & 41 & $100.0 \%$ & 81 & $100.0 \%$ \\
\multirow{2}{*}{$\begin{array}{l}\text { Post-operative } \\
\text { hearing }\end{array}$} & Mild & 0 & $.0 \%$ & 2 & $4.9 \%$ & 2 & $2.5 \%$ \\
& Moderate & 5 & $12.5 \%$ & 5 & $12.2 \%$ & 10 & $12.3 \%$ \\
& Normal & 35 & $87.5 \%$ & 34 & $82.9 \%$ & 69 & $85.2 \%$ \\
Hearing & Total & 40 & $100.0 \%$ & 41 & $100.0 \%$ & 81 & $100.0 \%$ \\
Gain (HG) & Excellent & 3 & $7.5 \%$ & 1 & $2.4 \%$ & 4 & $4.9 \%$ \\
& Good & 31 & $77.5 \%$ & 33 & $80.5 \%$ & 64 & $79.0 \%$ \\
& Poor & 6 & $15.0 \%$ & 7 & $17.1 \%$ & 13 & $16.0 \%$ \\
\hline
\end{tabular}


Table 3: Post-operative Cosmesis

\begin{tabular}{|c|c|c|c|c|c|c|c|}
\hline & & \multicolumn{2}{|c|}{ Case } & \multicolumn{2}{|c|}{ Control } & \multicolumn{2}{|c|}{ Total } \\
\hline \multirow{3}{*}{$\begin{array}{l}\text { Numbness } \\
\text { over pinna }\end{array}$} & Absent & 40 & $100.0 \%$ & 40 & $97.6 \%$ & 80 & $98.8 \%$ \\
\hline & Present & 0 & $.0 \%$ & 1 & $2.4 \%$ & 1 & $1.2 \%$ \\
\hline & Total & 40 & $100.0 \%$ & 41 & $100.0 \%$ & 81 & $100.0 \%$ \\
\hline \multirow{3}{*}{$\begin{array}{l}\text { Pain over } \\
\text { incision }\end{array}$} & Absent & 39 & $97.5 \%$ & 39 & $95.1 \%$ & 78 & $96.3 \%$ \\
\hline & Present & 1 & $2.5 \%$ & 2 & $4.9 \%$ & 3 & $3.7 \%$ \\
\hline & Total & 40 & $100.0 \%$ & 41 & $100.0 \%$ & 81 & $100.0 \%$ \\
\hline \multirow{3}{*}{$\begin{array}{l}\text { Change in } \\
\text { orientation } \\
\text { of pinna }\end{array}$} & Absent & 40 & $100.0 \%$ & 38 & $92.7 \%$ & 78 & $96.3 \%$ \\
\hline & Present & 0 & $.0 \%$ & 3 & $7.3 \%$ & 3 & $3.7 \%$ \\
\hline & Total & 40 & $100.0 \%$ & 41 & $100.0 \%$ & 81 & $100.0 \%$ \\
\hline \multirow{5}{*}{$\begin{array}{l}\text { Cosmetic } \\
\text { satisfaction }\end{array}$} & Good & 5 & $12.5 \%$ & 1 & $2.4 \%$ & 6 & $7.4 \%$ \\
\hline & Average & 17 & $42.5 \%$ & 0 & $.0 \%$ & 17 & $21.0 \%$ \\
\hline & Satisfactory & 17 & $42.5 \%$ & 37 & $90.2 \%$ & 54 & $66.7 \%$ \\
\hline & Poor & 1 & $2.5 \%$ & 3 & $7.3 \%$ & 4 & $4.9 \%$ \\
\hline & Total & 40 & $100.0 \%$ & 41 & $100.0 \%$ & 81 & $100.0 \%$ \\
\hline
\end{tabular}

\section{DISCUSSION}

The study done by A. C. Jyothi et al. showed $91.67 \%$ graft take up rate seen in endoscopic group when compared to $93.3 \%$ in the microscopic group ${ }^{[9]}$, showing that both the techniques were equal in terms of graft uptake. Another study done by N. Ahilasamy et al. showed that by the end of the follow up period of six months, 57(95\%) patients who underwent endoscopic tympanoplasty had an intact graft while 3 patients $(5 \%)$ had residual perforation. ${ }^{[10]}$ Another study done by Ying-Chieh Hsu et al. came to a conclusion that rates of complete healing of the tympanic membrane were not statistically significant $(p<0.05)$ between the endoscopic and microscopic tympanoplasty. ${ }^{[11]}$

Our studies showed similar results with $85 \%$ graft take-up in endoscopic tympanoplasty (ET) and $82.9 \%$ take-up in Microscopic tympanoplasty (MT), showing no statistical significance between the two techniques.

In a study done by Rakesh Kumar Maran et al., the endoscopic myringoplasty pre-operative and post-operative Air-Bone Gaps were $22.05 \pm 2.04$ and $9.05 \pm 1.36 \mathrm{~dB}$ respectively, and $21.81 \pm 1.85$ and $8.55 \pm 1.44 \mathrm{~dB}$ in microscopic myringoplasty respectively. The $\mathrm{P}$ value was 1.752 showing no significant difference between them for hearing improvement. ${ }^{[12]}$ Another study done Ying-Chieh Hsu et al. also had a similar result. The average hearing gain in the trans endoscopic ear surgeries (TEES) group was $10.3 \pm 6.4 \mathrm{~dB}$ and in the microscopic ear surgeries (MES) group was $12.4 \pm 7.5 \mathrm{~dB}(p=0.1663)$. There was no statistically significant difference between the two groups with respect to average of pre- and postoperative $\mathrm{ABG}$ or hearing gain. ${ }^{[11]}$
In our study post-operative hearing gain in endoscopic group was good in $31(77.5 \%)$ patients and excellent in $3(7.5 \%)$ patients and poor in $6(15 \%)$ patients. In microscopic group, it was good in $33(80.5 \%)$ and excellent in $1(2.4 \%)$ and poor in $7(17.1 \%)$ patients, showing no difference in postop hearing gain between the ET and MT groups.

The study done by G. Shaul Hameed et al. showed a graft uptake of $90 \%$ with temporalis fascia and $80 \%$ graft uptake with tragal perichondrium. Postoperatively in $70 \%$ patients, air conduction gain was up to $21-30 \mathrm{~dB}$ in temporalis fascia group and $60 \%$ in tragal perichondrium group. ${ }^{[13]}$

Our study had similar results: graft uptake was $85.0 \%$ in endoscopic tympanoplasty (ET) using tragal perichondrium (TP) and $82.9 \%$ in microscopic Tympanoplasty (MT) using Temporalis fascia (TF). This showed that the tragal perichondrium was an equally efficient graft material when compared to temporalis fascia.

A. C. Jyothi et al. showed that in patients who had anatomical variations of the ear canal, the entire tympanic membrane was visualised better in endoscopic technique; however, microscopic group had better depth perception. ${ }^{[9]}$ The disadvantage of endoscopic procedure was that a small amount of blood could obscure the vision. Hence proper haemostasis is a must. However, this problem could be tackled in microscopic technique with the aid of a suction as one hand was free to operate the suction tip. Studies done by G. Lakpathi et al. showed a similar experience where bleeding in endoscopic technique obscured 
their vision and required meticulous haemostasis ${ }^{[14]}$ In a similar study done by Rakesh Kumar Maran et al., after refreshing edges, visualization of perforation margins in large and subtotal perforation was good in endoscopic procedure. Furthermore, incudo-stapedial joint, eustachian tube orifice and round window were better visualised in endoscope, and curvy external auditory canals were easily negotiated with endoscope. In microscopic technique, the patient's head required frequent adjustment. Curettage and canaloplasty were not required in endoscopic procedure, but if overhang caused difficult visualization of ossicular chain it was required in microscopic procedure. In their study canaloplasty was done in 2 patients and curettage in 2 patients $^{[12]}$.

In our study, $97.5 \%$ of the patients from ET had good satisfaction of clarity of view (SCV) while $2.5 \%$ had average SCV due to bleeding, while in the MT group $100 \%$ had good SCV, showing no statistical difference between the two groups. There was no difference in satisfaction of depth perception (SDP) between the ET (97.5\%) and MT groups (82.8\%). Entire tympanic membrane visualization (ETM) was better in endoscopic group (92.5\%) compared to microscopic group (43.9\%) and $p$ value $(0.0)$ was highly significant showing that ETM was better visualised in the endoscopic group.

In the study done by A. C. Jyothi et al., the average time taken for surgery in endoscopic group was 1 hour when compared to the microscopic group which was 2 hours 9. Another study done by N. Ahilasamy et al. showed that the operative time taken with endoscopic technique for most of the patients (70\%) was 30-45 minutes. While in the rest of the patients (30\%), surgery was completed by $60 \mathrm{~min}$, no surgery took more than 1 hour 10 . A similar study done by Rakesh Kumar Maran et al. showed time taken for surgery was less in endoscopic myringoplasty compared to microscopic technique, as post aural soft tissue work was not required. This therefore shortened operative time and caused lesser surgical trauma, leading to fast post-operative recovery. The average operative duration was $65.5 \pm 3.45$ minutes in endoscopic group, and $85.7 \pm 3.42$ minutes in microscopic group. The operative duration was significantly lower in ET, with a p value of $0.001^{[12]}$.

In our study, in ET group the minimum time taken for surgery was $45 \mathrm{~min}$ and maximum time taken was $1 \mathrm{~h} 20 \mathrm{~min}$. Average time taken was $62.5 \mathrm{~min}$. In the MT group, minimum amount of time taken was $1 \mathrm{~h}$ and maximum time for surgery was $2 \mathrm{~h} 30 \mathrm{~min}$ and average time was $1 \mathrm{~h} 55 \mathrm{~min}$. The $p$ value was highly significant $(p=0.00)$, showing significantly shorter duration for surgery for endoscopic technique compared to the microscopic group. The time saved during surgery was mainly due to a smaller incision and closure time in endoscopic technique, compared to the microscopic technique. This was because endoscopic technique required a smaller $2 \mathrm{~cm}$ incision compared to the $5 \mathrm{~cm}$ incision required for the microscopic technique. In addition, microscopic technique required closure of the incision, whereas there was no closure required for the endoscopic technique; the incision on the tragus in ET was simply left to close on its own by placing a cotton ball in the external auditory canal behind the tragus.

The study done by G. Lakpathi et al., stated that they got better cosmetic results by avoiding the postauricular incision in endoscopic group which reduced the chance of auricular displacement and asymmetry of pinna. Cosmetic result was rated as excellent in all the patients of the endoscope group at the end of 6 months, whereas in the microscope group, $6(20 \%)$ patients rated their cosmetic result as poor, $15(50 \%)$ rated it as satisfactory while $9(30 \%)$ patients rated an excellent cosmetic result. ${ }^{[14]}$

Our studies showed a better cosmetic satisfaction in endoscopic group with $12.5 \%$ showing good satisfaction, $42.5 \%$ patients with average verdicts, compared to the microscopic group where 90.2\% patients had satisfactory and $2.4 \%$ had good verdicts. This was because post auricular incision was avoided in ET group which did not require dissection and closure. Cosmetic satisfaction was highly significant with $p$ value of 0.00 .

In a study done by Rakesh Kumar Maran et al., Wong - Baker FACES pain rating scale was used for the purpose of recording post-operative pain till the discharge date. Patients were asked about post-operative pain one day after surgery; in endoscopic patients there was mild to no pain while in the microscopic patients, there was significant and irritating pain due to the post aural route. 12 Furthermore, Coskun et al stated that, endoscopic myringoplasty gave excellent cosmetic results as the post aural scar was avoided. ${ }^{[15]}$

In our study, in ET group one female patient presented post-operatively with pain over incision site (POI). Examination revealed an exposed cartilage with some granulation around the incision site, but there was no issue in relation to graft uptake. This was resolved easily in the outpatient department, where under local anaesthesia the exposed cartilage was resected out and incision site was sutured together with no negative outcomes on subsequent follow up. No patients in ET had any numbness over the pinna (NOP) or change in orientation of pinna (CIO) and overall cosmetic satisfaction (CS) was good. 
On the other hand, in MT group, one patient had pain over incision site, three patients had numbness over pinna and three patients had change in orientation of the pinna, but the overall cosmetic satisfaction was satisfactory. Our study showed that for overall CS, ET had better outcomes than the MT group and these results were highly significant with a $\mathrm{p}$ value of 0.00 .

\section{CONCLUSION}

Through our study, we concluded that the endoscope delivers a better visualization of the entire tympanic membrane without the need to manipulate the patients head. There was no difference in clarity of view, depth of perception, round window reflex elicitation, ossicular chain visualisation, graft uptake or hearing gain between the two procedures in our study, showing that endoscopic technique was equally effective when compared to microscopic technique. Tragal perichondrium was as efficient a graft material as temporalis fascia. Microscopic technique required a long post auricular incision, dissection and closure which led to change in normal orientation of the pinna, pain over incision site and poor cosmetic satisfaction. The post auricular dissection and closure of incision site also extended the duration of surgery which was not required in endoscopic technique, which led to significant reduction of duration of surgery.

\section{CONFLICT OF INTEREST}

There are no conflicts of interest.

\section{REFERENCES}

1. El-Guindy. A Endoscopic transcanal myringoplasty. J Laryngol Otol. 1992;(106):493-495.

2. Khan MM, Parab SR. Endoscopic cartilage tympanoplasty: A two-handed technique using an endoscope holder. Laryngoscope. 2016;126(8):18931898.

3. Himani Lade, Santosha Ram Choudhary AV. Endoscopic vs microscopic myringoplasty: a different perspective. Eur Arch Oto-Rhino-Laryngology. 271(7):1897-1902.

4. Jahner JA, Schlauch RA, Doyle T. A comparison of American Speech-Language Hearing Association guidelines for obtaining speech-recognition thresholds. Ear Hear [Internet]. 1994 Aug;15(4):324329. Available from: http://www.ncbi.nlm.nih.gov/ pubmed/7958531

5. Choi N, Noh Y, Park W, Lee JJ, Yook S, Choi JE, et al. Comparison of endoscopic tympanoplasty to microscopic tympanoplasty. Clin Exp Otorhinolaryngol. 2017;10(1):44-49.

6. Anoop Raj; Rajiv Meher 2 S. Endoscopic Transcanal Myringplasty - A study. Indian J Otolaryngol Head Neck Surg. 2001;53(1): 47-49.

7. J. M. Kartush. "Ossicular Chain Reconstruction. Capitulum to Malleus,." Otolaryngol Clin North Am. 1994; 27(4):689-715.

8. A. S. Harugop, R. S. Mudhol RAG. A Comparative Study of Endoscope Assisted Myringoplasty and Microscopic Myringoplasty. Indian J Otolaryngol Head Neck Surg. 2008;60:298-302.

9. Jyothi AC, Shrikrishna BH, Kulkarni NH, Kumar A. Endoscopic Myringoplasty Versus Microscopic Myringoplasty in Tubotympanic CSOM: A Comparative Study of 120 Cases. Indian J Otolaryngol Head Neck Surg. 2017;69(3):357-362.

10. Ahilasamy N, Shanti B, Rajasekaran S. Endoscopic Tympanoplasty Using Nasal Septal Cartilage Allograft. Indian J Otolaryngol Head Neck Surg. 2017;69(2):199-203.

11. Hsu YC, Kuo CL, Huang TC. A retrospective comparative study of endoscopic and microscopic Tympanoplasty. J Otolaryngol - Head Neck Surg. 2018;47(1):1-7.

12. Maran RK, Jain AK, Haripriya GR, Jain S. Microscopic Versus Endoscopic Myringoplasty: A comparative study. Indian J Otolaryngol Head Neck Surg [Internet]. 2018; Available from: http://link. springer.com/10.1007/s12070-018-1341-4.

13. Hameed GS, Prasad BD, Babu PR, Care P. A Comparative Study of Temporalis Fascia versus Tragal Peri- chondrium as Graft in Repair of Tympanic Membrane Perforation. Indian J Mednodent Allied Sciences. 2015;3(3):150-154.

14. Lakpathi G, Sudarshan Reddy L, Anand. Comparative Study of Endoscope Assisted Myringoplasty and Microscopic Myringoplasty. Indian J Otolaryngol Head Neck Surg. 2016;68(2):185-190.

15. Berna Uslu Coskun, Ugur Cinar, Huseyi Seven, Seher Ugur BD. The effects of the incision types in myringoplasty operations on cosmesis. Eur Arch Oto-Rhino-Laryngology Head Neck.2006; 263(9):820-822. 